\title{
Suppression of aquaporin, a mediator of water channel control in the carcinogenic liver fluke, Opisthorchis viverrini
}

\author{
Sirikanda Thanasuwan ${ }^{1}$, Supawadee Piratae ${ }^{1}$, Paul J Brindley², Alex Loukas³ ${ }^{3}$ Sasithorn Kaewkes ${ }^{1}$ \\ and Thewarach Laha ${ }^{1,4^{*}}$
}

\begin{abstract}
Background: Opisthorchiasis and Opisthorchis viverrini-associated bile duct cancer represent major public health threats in Thailand and Laos. The tegument of this food borne fluke plays pivotal roles in parasite metabolism, homeostasis and osmoregulation. Excretory/secretory products also pass from the fluke to the biliary environment, products that likely underlie pathogenesis of liver fluke infection. Aquaporins (AQPs), belong to the major intrinsic protein superfamily of integral plasma membrane channel proteins that selectively transport water across cell membranes. AQPs play key roles as water and ion transport channels through the tegument of helminth parasites.

Methods: Here, two forms of AQP mRNAs from the adult developmental stage of $O$. viverrini, termed $O$. viverrini aquaporin-1 and -2 (Ov-aqp-1 and -2) were investigated. Roles of Ov-aqp-1 and -2 in the movement of water across the tegument of this carcinogenic liver fluke were investigated using RNA interference.

Results: OV-AQP-1 and Ov-AQP-2 contain unique characteristic asparagine-proline-alanine (NPA) motifs of AQP transmembrane proteins. Phylogenetic analysis indicated that Ov-AQPs belong to an expanding group of aquaglyceroporin-like water channel proteins characterized from helminth and protozoan parasites, which is pivotal to the specialized requirements of water and solute control during parasitism. Elevated transcription of Ov-aqp-1 was evident in the egg, cercaria, metacercaria and adult stages of $\mathrm{O}$. viverrini, whereas Ov-aqp-2 transcripts were detected at higher level in egg, metacercaria, cercaria and adult stage, respectively. RNA interference using electroporated dsRNA suppressed transcript levels of Ov-aqp-1 and Ov-aqp-2 in adult worms by 58-99\% over periods of up to 16 days in vitro. Suppression of Ov-aqp-1 and Ov-aqp-2 in vitro disabled water transport in adult flukes.

Conclusion: The apparently pivotal roles of Ov-AQP in solute homeostasis at the fluke surface suggest that deeper investigation will be informative for the pathophysiology of $O$. viverrini, and may uncover intervention targets, particularly in view of the singularly notable predilection of this pathogen for residence within ducts of the biliary tree.
\end{abstract}

Keywords: O. viverrini, Cholangiocarcinoma, RNA interference, Aquaporin, Liver fluke, Tegument

\footnotetext{
* Correspondence: thewa_la@kku.ac.th

${ }^{1}$ Department of Parasitology, Faculty of Medicine, Khon Kaen University,

Khon Kaen 40002, Thailand

${ }^{4}$ Liver Fluke and Cholangiocarcinoma Research Center, Faculty of Medicine,

Khon Kaen University, Khon Kaen 40002, Thailand

Full list of author information is available at the end of the article
}

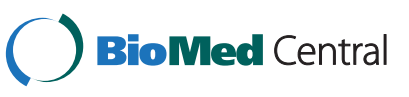

(C) 2014 Thanasuwan et al.; licensee BioMed Central Ltd. This is an Open Access article distributed under the terms of the Creative Commons Attribution License (http://creativecommons.org/licenses/by/4.0), which permits unrestricted use, distribution, and reproduction in any medium, provided the original work is properly credited. The Creative Commons Public Domain Dedication waiver (http://creativecommons.org/publicdomain/zero/1.0/) applies to the data made available in this article, unless otherwise stated. 


\section{Background}

Opisthorchis viverrini is highly endemic in Southeast Asia, where more than 10 million people are infected $[1,2]$. Studies with hamster models of both chronic O. viverrini infection and exposure to nitrosamines have determined that host responses are key factors in carcinogenesis of liver fluke-induced cholangiocarcinoma [3]. Membrane proteins of O. viverrini without orthologues/paralogues in mammals are of particular interest for the development of vaccines and drugs, because the absence of host homologues raises therapeutic margins of safety [4-6].

AQPs are the major intrinsic protein (MIP) of integral plasma membrane channel proteins that are passively permeated by water and small, uncharged solutes $[7,8]$. AQPs have been identified based on their highly conserved dual asparagine-proline-alanine (NPA) boxes, critical for the formation of a water-permeating pore. The sequences of conventional AQPs have only minimal or modest identity to one another but they share conserved six transmembrane domains and hydrophobic NPA box-like repeats $[9,10]$. Moreover, some AQP-like sequences exhibit only poor sequence conservation for the NPA motifs [11]. The NPA motifs of AQPs play crucial roles for movement of water across cell membranes [8].

AQPs have been investigated in several parasites and observed to provide key functions in transport of water and other small solutes. Moreover, AQPs facilitate and inhibit uptake of lactate and other anthelmintics [12-14]. In schistosomes, AQP is a major tegument protein with functions in trans-tegumental water movement, absorption of nutrients and other metabolites [14,15]. AQPs are highly expressed in the transcriptomes and tegumental proteome of $O$. viverrini $[16,17]$. In this study, discrete mRNA sequences encoding two AQP-like transporters were isolated from a cDNA library established from the adult stage of O. viverrini. The properties of these water channel transporters were investigated using bioinformatics approaches, phylogenetic analyses and gene silencing via RNA interference to highlight the physiological roles of AQPs in liver fluke biology and parasitism.

\section{Methods}

\section{Opisthorchis viverrini}

Metacercariae of $O$. viverrini were collected from the flesh of naturally infected cyprinid fish from Khon Kaen province, Thailand by digestion with $0.25 \%$ pepsin, as described [18]. Syrian golden hamsters, Mesocricetus auratus, purchased from the Animal Unit, Faculty of Medicine, Khon Kaen University were infected with 50 metacercariae by orogastric gavage [19]. Hamsters were maintained at the animal facility, Faculty of Medicine, Khon Kaen University using protocols approved for animal experimentation by the Animal Ethics Committee of Khon Kaen University, based on the Ethics of Animal Experimentation of the National Research Council of Thailand (Approval number AEKKU43/2555). Hamsters were euthanized six weeks after infection, when adult flukes were recovered from bile ducts and the gall bladder. The worms were rinsed in sterile $0.9 \% \mathrm{NaCl}$ to remove residual host cells and debris.

Eggs of $O$. viverrini were collected from worms recovered from euthanized hamsters; flukes were maintained in RPMI media containing antibiotics (streptomycin/penicillin, $100 \mu \mathrm{g} / \mathrm{ml}$ ) at $37^{\circ} \mathrm{C}$, in an atmosphere of $5 \% \mathrm{CO}_{2}$ and incubation for $18 \mathrm{~h}$ [20]. Eggs were collected by centrifugation at $5,241 \mathrm{~g}$ for $10 \mathrm{~min}$ and stored at $-70^{\circ} \mathrm{C}$. Cercariae of $O$. viverrini were shed from naturally infected Bithynia sp. snails collected in farmlands in Khon Kaen province [21]. Snails were placed into plastic containers filled with de-chlorinated water, 4-5 snails per container, and exposed to the light for $2 \mathrm{~h}$, after which cercariae were collected by centrifugation of the supernatant water at $5,241 \mathrm{~g}, 10 \mathrm{~min}$.

\section{Isolation of aquaporin genes from $O$. viverrini}

cDNA sequences encoding full open reading frames (ORFs) of $O v-a q p-1$ and $O v$ - $a q p$ - 2 were amplified by PCR from a cDNA library of transcripts from the adult developmental stage of the fluke [16]. The specific primers for PCR amplification of the $O v-a q p$ genes were designed based on expressed sequence tags (ESTs) and EST contigs. cDNA sequences encoding full length ORFs of $O v-a q p-1$ (GenBank accession EL618688) [16] and Ov-aqp-2 (OV_ contiq1681, available at http://bioinfosecond.vet.unimelb. edu.au/) [22] were identified from our previous transcriptomics study. The primers for Ov-AQP-1 F were 5'-AG CATGGCTGGTAGTCTCTCATC and Ov-AQP-1R5'-AG CGGATCCTCAGTTTTTTTTCTGGCG. The primers of $O v$-AQP-2 F were 5'-AGCCATATGATGAGTTTGGAA TGCGAAAC and Ov-AQP-2R5'-AGCGGATCCCTAGG CAAGCAGTTCAGTTC. PCR reaction mixes included the O. viverrini cDNA library (100 ng), $0.2 \mathrm{mM}$ dNTP, $1.5 \mathrm{mM}$ $\mathrm{MgCl}_{2}$ with 1 unit Taq polymerase (Invitrogen, USA). Amplification was accomplished with 35 cycles of denaturation at $95^{\circ} \mathrm{C}$ for $1 \mathrm{~min}$, annealing at $60^{\circ} \mathrm{C}$ for $1 \mathrm{~min}$, extension at $72^{\circ} \mathrm{C}$ for $2 \mathrm{~min}$ and a final extension at $72^{\circ} \mathrm{C}$ for $10 \mathrm{~min}$. The amplicons were separated and sized by electrophoresis through agarose followed by staining with ethidium bromide. Products of interest were isolated from the gel using a kit (GeneJET ${ }^{\mathrm{mm}}$ gel extraction, Fermentas, EU), ligated into the pGEM-T Easy vector (Promega, USA), after which ligation products were used to transform Escherichia coli strain JM109 competent cells (Promega). Plasmids isolated from the resulting colonies were sequenced using BigDye terminator method $\left(1^{\text {st }}\right.$ BASE, Singapore); sequences were analyzed using Blast search 
against GenBank databases [23] and compared to consensus sequences of $O v$-AQPs from ESTs of $O$. viverrini $[16,22]$. Recombinant plasmids containing cDNA sequences encoding the entire ORFs of $O v-a q p-1$ and $O v$ $a q p-2$ were termed $p O v-a q p-1$ and $p O v-a q p-2$.

\section{Sequence and phylogenetic analyses}

Nucleotide sequences and chromatograms were evaluated using BioEdit V7.0.5 [24]. The edited sequences were translated to ORFs with assistance of software at http:// bio.lundberg.gu.se/edu/translat.html. Signal peptides from the deduced amino acid sequences were predicted and analyzed by SignalP 3.0 Server at http://www.cbs.dtu.dk/ services/SignalP/. Transmembrane helices were predicted by using TMHMM Server v. 2.0 (http://www.cbs.dtu.dk/ services/TMHMM-2.0/). N-Glycosylation sites were predicted by using the NetNGlyc 1.0 Server at http://www. cbs.dtu.dk/services/NetNGlyc/. Other divergent sequences were compared and multiple alignments constructed by ClustalW in the BioEdit program [24]. ORFs of $O v$-AQPs were aligned with well characterized AQP protein sequences from informative species using ClustalW in the BioEdit program [24]. A phylogenetic tree was constructed with p-distance matrix using the neighbor-joining method [25] with 1,000 bootstrap samplings in the MEGA version $6.06[26]$.

\section{RNA extraction and quantitative real time reverse transcription PCR}

Real time, reverse transcription PCR was undertaken to monitor the expression of the $O v-a q p$ genes during the developmental cycle of the liver fluke - egg, cercaria, metacercaria and adult stages were examined, and at intervals after exposure by electroporation to dsRNA. Briefly, total RNA was extracted from the developmental stages of the parasite using TRIZOL (Invitrogen). Any residual DNA remaining in the RNA preparations was removed by DNase digestion. Double stranded cDNA was synthesized from equal amounts of total RNA template $(1 \mu \mathrm{g})$ using a cDNA synthesis kit (Fermentas). Quantitative real-time PCR was performed using custom SYBR Green Assays. The primers to detect $O v-a q p-1$ (spanning coding DNA positions 3-280), were AQP1_EXF, 5'-GG CTGGTAGTCTCTCATC-3' and AQP1_EXR, 5' -CGTA TCCCATAGTACCGCTG-3'. Ov-aqp-2 transcripts (spanning nt positions 16 to 256) were designated Ov-AQP2 EXF: 5'-GAAACCCGATTTCGAAGAGG and Ov-AQ P2_EXR: 5'-TGATCCCGGAGAAGAATACG. PCRs were performed in triplicate using SYBR Green reagents and a thermal cycler with a real time detector (ABI 7500); SYBR Green reactions were prepared by adding $12.5 \mu \mathrm{l}$ of SYBR Green Master Mix (TAKARA Perfect Real-time Kit, Japan), $0.5 \mu \mathrm{l}(10 \mathrm{mM})$ of forward primer and reverse primers, $0.5 \mu \mathrm{l}$ of reference dye (ROX), $1 \mu \mathrm{l}$ (equivalent to
50 ng of total RNA) of first-stand cDNA and water to a final volume of $25 \mu \mathrm{l}$. The thermal cycling conditions used were: initiation pre-heat for one cycle at $95^{\circ} \mathrm{C}, 10 \mathrm{~min}$; 40 cycles of denaturation at $95^{\circ} \mathrm{C}, 30 \mathrm{sec}$; annealing at $55^{\circ} \mathrm{C}, 30 \mathrm{sec}$; extension at $72^{\circ} \mathrm{C}, 45 \mathrm{sec}$. Expression levels of the Ov-aqp-1,Ov-aqp-2and actin mRNAs (OvAE1657, GenBank EL620339) were determined as described [20].

To determine the extent of gene silencing induced by dsRNAs, the mRNA expression levels of $O v-a q p-1$ and $O v-a q p-2$ (or firefly luciferase as an irrelevant control, below) were normalized with actin mRNA and presented as the unit value of $2^{-\Delta \Delta \mathrm{Ct}}$ where $\Delta \Delta \mathrm{Ct}=\Delta \mathrm{Ct}$ (treated worms) - $\Delta \mathrm{Ct}$ (non-treated worms) [20,27]. Data are presented as the mean \pm 1 standard deviation. Differences between groups were assessed using Student's $t$-test (GraphPad Prism Software); $p$ values of $\leq 0.05$ were considered statistically significant.

\section{Preparation and delivery of dsRNA}

dsRNAs were designed to span 605 nt of $O v$-aqp-1 (full ORF spanning nt193-798) and 575 nt of $O v-a q p-2$ (full ORF spanning nt 201-776). Target sequences were amplified from plasmids $p O v$-aqp-1 and $p O v$-aqp-2 (above) using primers flanked with a T7 RNA polymerase promoter sequence, indicated in underlined italic bold faced, at the $5^{\prime}$ end. $O v$-aqp1 was generated using primers ds-aqp1_T7-F, 5'-TAATACGACTCACTATAGGGGGT AGCAACGTCTCGGCTand ds-aqp1_T7-R, 5' -TAATAC GACTCACTATAGGGGTAGAGTAACACTCCGAG. Ov$a q p-2$ was generated using primers ds-aqp2_T7-F, 5'TAATACGACTCACTATAGGGAGTGAGTCTTGGCTG GGGTA and ds-aqp2_T7-R, 5'-TAATACGACTCACTA TAGGGTACGGTCCGACGATTGGTAT. dsRNAs were synthesized using a MEGAscript RNAi Kit (Ambion, USA). The irrelevant negative control, luciferase (LUC) dsRNA was constructed from plasmid pGL3-basic (Promega), because this sequence does not match any targets in the $O$. viverrini genome. It was amplified using primers ds-LUC_T7-F5' -TAATACGACTCACTATAGGGTGCGC CCGCGAACGACATTTA and ds-LUC_T7-R5'-TAATA CGACTCACTATAGGGGCAACCGCTTCCCCGACTTC CTTA [28]. The thermal cycling conditions were 35 cycles of denaturation at $94^{\circ} \mathrm{C}, 30 \mathrm{sec}$; annealing at $55^{\circ} \mathrm{C}, 30 \mathrm{sec}$; and extension at $72^{\circ} \mathrm{C}, 60 \mathrm{sec}$. Amplicons were sized by electrophoresis and purified, as above. Concentrations of dsRNA were determined by spectrophotometer (NanoVue, GE Healthcare, USA).

To deliver $O v$-aqp dsRNAs, adult worms (30 per treatment group) were washed with sterile phosphate buffer, then transferred to a cuvette, $4 \mathrm{~mm}$ gap (Bio-Rad) in $100 \mu \mathrm{l}$ electroporation buffer (RPMI-1640, 1x antibiotic/antimycotic, $1 \%$ glucose, $1 \mathrm{mM}$ trans-epoxysuccinyl-L-leucylamido (4-guanidino) butane (E64) (Sigma) containing $50 \mu \mathrm{g} O v-a q p-1, O v-a q p-2$ or luc dsRNAs. Each group of 
worms was subjected to square wave electroporation using a single $20 \mathrm{~ms}$ impulse at $125 \mathrm{~V}$ (Gene PulserXcell, Bio-Rad, USA). Subsequently, worms were maintained in RPMI culture medium (one $\mathrm{ml}$ culture medium in each well of a 24 well plate) supplemented with $2 \mathrm{mg} / \mathrm{ml}$ of dsRNA at $37^{\circ} \mathrm{C}$ under $5 \% \mathrm{CO}_{2}$ in air. Similarly treated worms in electroporation buffer without dsRNA served as controls. Worms were soaked in $2 \mathrm{mg}$ dsRNA for 16 days $(O v-a q p-1)$ and 7 days $(O v$-aqp-2) with changes of media containing dsRNA every second day. Worms were collected for analysis on days 1, 3, 6, 10 and 16 for $O v$-aqp-1 and on days 1,3 and 7 for $O v$-aqp-2 following electroporation.

\section{Water transport assay and size measurements of parasites}

Water transportation in normal worms was investigated to obtain determine optimal times for observation of the transformed worms. The volume of water transport via the body wall of adult flukes was investigated by monitoring swelling of the flukes after exposure to water. Wild type adults were incubated in distilled water for $5,10,30$, 60 and $120 \mathrm{~min}$. Photomicrographs were captured on these cultures, after which surface area of the flukes was measured using the NIS-Elements D 3.22.00 software (Build 710) - Driver selection (Nikon, Japan). The function of the O. viverrini AQPs as water channels was investigated using RNAi to knock down the genes after treatment with $O v-a q p-1, \quad O v-a q p-2$ and $O v-a q p-1+O v-a q p-2$ dsRNAs. Water transportation through the tegument of control and dsRNA-treated worms was determined by measuring the surface area of the fluke after placing the worms in water for $10 \mathrm{~min}$. Briefly, worms electroporated with dsRNAs of $O v$-aqp or luc were cultured in complete RPMI medium for $24 \mathrm{~h}$ prior to transfer to hypo-osmotic medium (distilled water) for $10 \mathrm{~min}$. Photomicrographs were recorded and the surface area of individual worms ascertained before and after exposure to the hypo-osmotic conditions. To compare surface area of the liver flukes before and after transfer, images were recorded so that the surface area of individual adult worms could be determined. The liver flukes were photographed before and $10 \mathrm{~min}$ after transfer into water, and the sizes of a sample of individual parasites from control and $O v-a q p$ suppressed groups were ascertained and compared.

\section{Histological examination}

To observe swelling of the worms, sections of fixed flukes after dsRNA treatment for 1, 3 and 7 days were examined. Briefly, the whole worms were fixed in hot $10 \%$ buffered formalin overnight then processed conventionally. Adult worms in paraffin sections were de-paraffinized in xylene three times, five minutes each. The $4 \mu \mathrm{m}$ thick sections were stained with hematoxylin and eosin. Thereafter, the sections were dehydrated, cleared and mounted. Histology of adult worms was compared and contrasted using light microscopy [29].

\section{Statistical analysis}

Means \pm standard deviations were calculated and presented using Microsoft Excel or GraphPad Prism software. Differences between groups were assessed using Student's $t$-test (GraphPad Prism Software, USA); $p \leq 0.05$ was considered statistically significant.

\section{Results}

\section{Sequences and structure of $O$. viverrini aquaporin}

The full length cDNA sequences encoding two isoforms of $O$. viverrini AQPs (Ov-AQPs) were designated $O v$ - $a q p$ 1 and $O v-a q p-2$; nucleotide sequences of $O v-a q p-1$ and $O v$-aqp-2 have been assigned GenBank accession numbers KF697690 and KF697691, respectively. Ov-aqp-1and $O v$-aqp-2 are 864 and 921 nt and encode 287 and 306 amino acid ORFs, respectively. Both $O v-\mathrm{AQP}-1$ and $O v-$ AQP-2 are predicted to contain six-transmembrane domains (TM1-6) connected by five loops (A-E) (Figure 1). Potential $N$-glycosylation sites are present at Asn-67 in $\mathrm{Ov}$-AQP-1 and Asn-293 in Ov-AQP-2 (Figure 1A). OvAQP-1 and $O v$-AQP-2 sequences contain conserved NPA motifs as found in other AQPs. Both the amino and carboxyl termini of $O v$-AQP-1 and $O v$-AQP-2 are predicted to be intracellular $O v$-AQP-1 consists of two hemipores, each hemipore containing three transmembrane domains (Figure 1B). The pore that forms in the AQPs is composed of two hemipores where loops $B$ and $E$ form the poreforming signature motif, NPA (Asn-Pro-Ala) via an overlap to form the characteristic pore between the membrane bilayer (Figure 1B). It is noteworthy that both of these two new water channel molecules include the aspartic acid residue (D) in the second NPA box that is a diagnostic sequence for aquaglyceroporins, a category of aquaporinlike water channel proteins discrete from the 'classical' aquaporins. Aquaporins sensu stricto are water selective or specific water channels, whereas the aquaglyceroporins are permeable to other small, uncharged molecules, in particular glycerol, in addition to water (Benga 2012).

\section{Phylogenetic analysis of Ov-AQP}

The phylogenetic relationships of $O v-\mathrm{AQP}-1$ and $O v$ AQP-2 protein sequences were investigated by comparison with well-characterized AQPs from various animals [9]. A neighbor-joining tree indicated that $O v$-AQPs formed a clade with AQPs from several invertebrates, including other trematodes, and also nematode and protozoan parasites such asaquaporin-3 from Clonorchis sinensis (GAA31414) [30] (Figure 2). Ov-AQP-2 formed a robust clade with aquaporin-9 from C. sinensis (GAA55320 and GAA55323) [30] and a group of aquaporins from the 


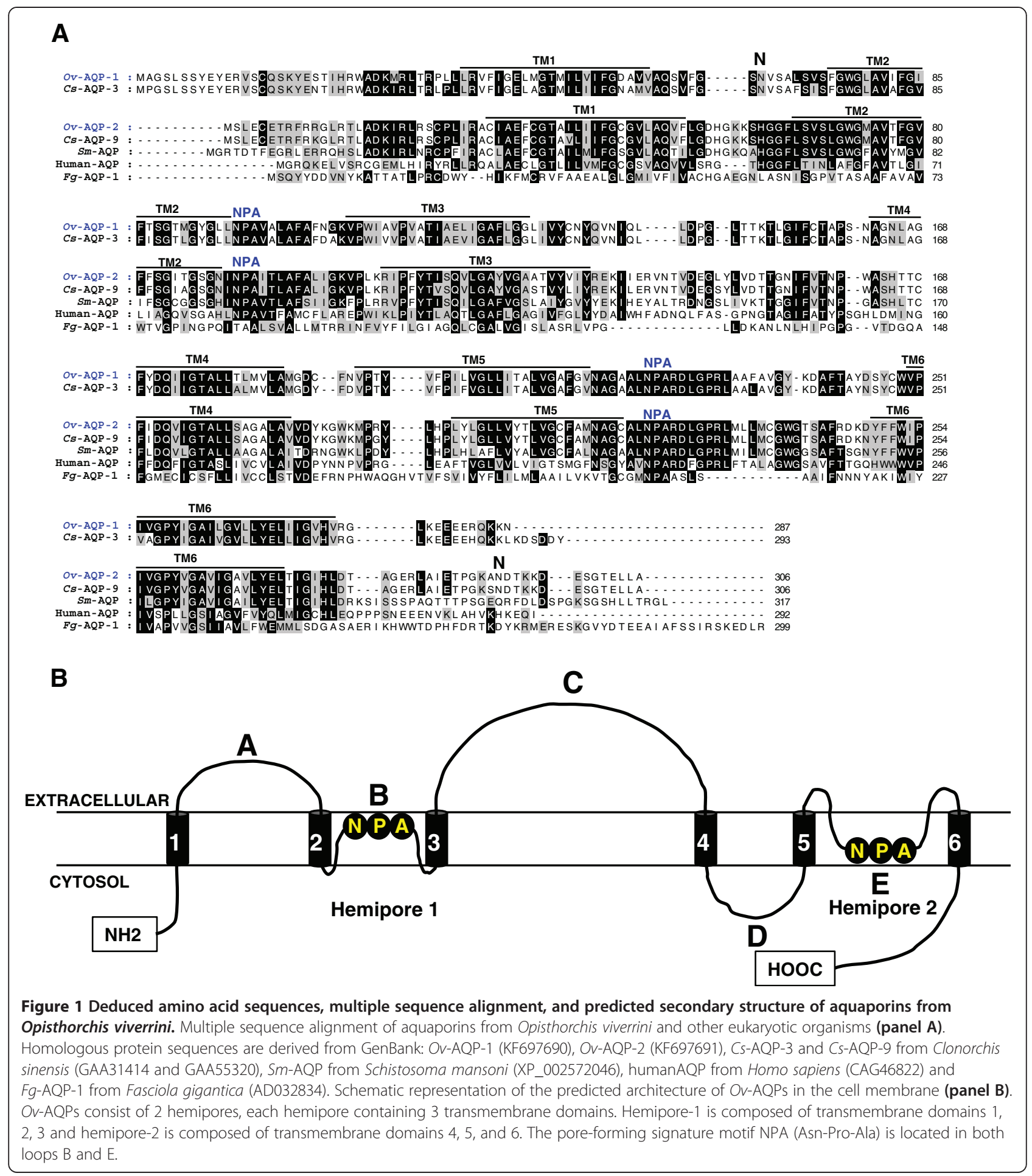

schistosomes (blood flukes) [12]. Ov-AQP-1 was less closely related to AQPs from other trematodes, including $O v$-AQP-2, branching at amore distant position in the phylogram along with a predicted AQP from $C$. sinensis, GAA31414). However, both new $O v$-AQP water channel proteins branched with the aquaglyceroporins rather than the 'classical' (strict) aquaporins.

\section{Developmental expression of Ov-aqp genes}

Levels of expression of $O v-a q p-1$ and $-a q p-2$ mRNAs relative to expression of the actin control gene were examined by qRT-PCR among developmental stages of $O$. viverrini. Expression of Ov-aqp-1 expression was seen in all developmental stages examined but was highest in metacercariae (Figure 3). Ov-aqp-2 expression was also 


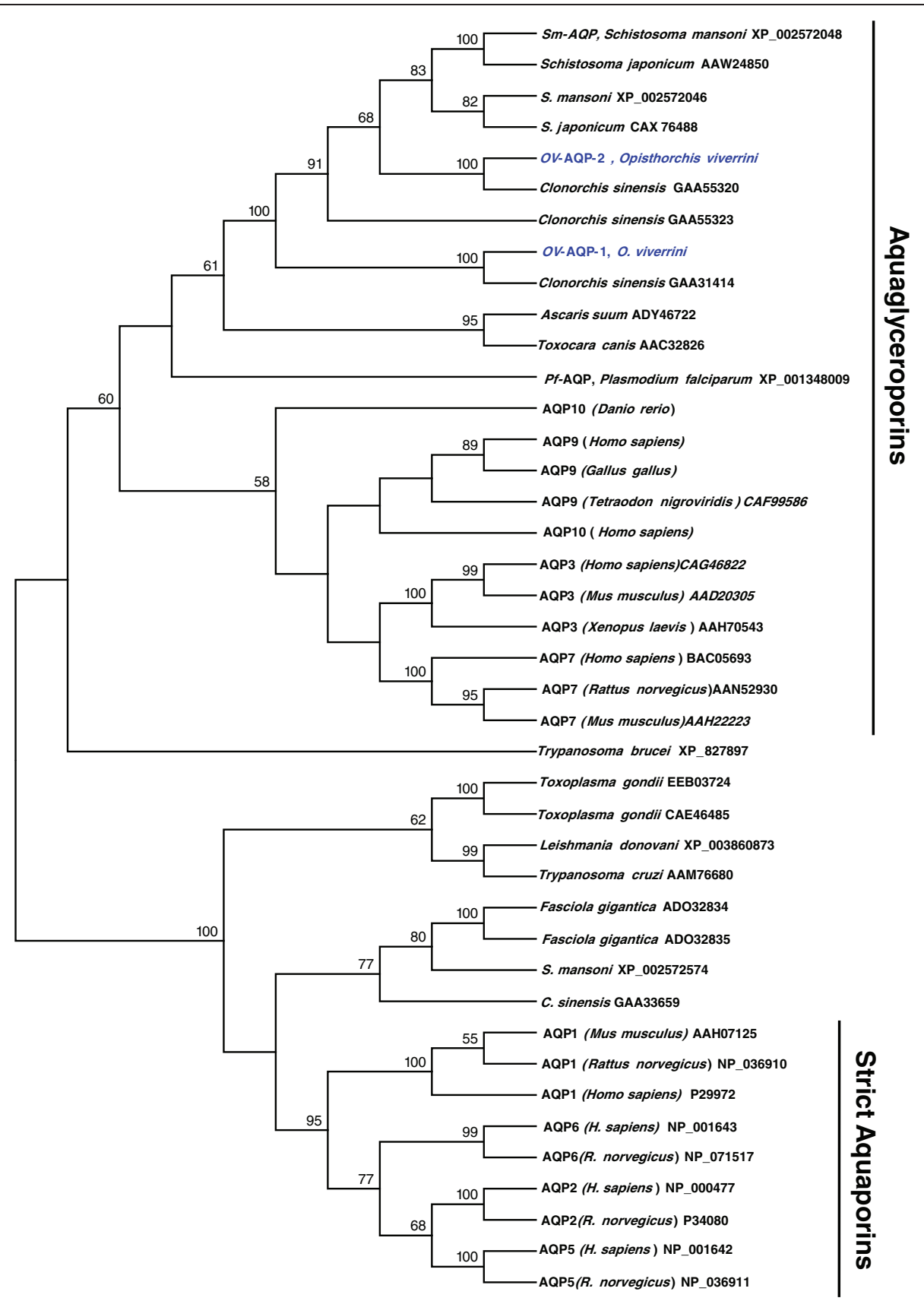

Figure 2 Phylogram constructed using the neighbor joining method to compare the relationship between the open reading frames of Ov-AQPs of Opisthorchis viverrini and homologues within the aquaporin superfamily. The accession numbers for sequences included in the phylogenetic analysis are shown in each operational taxonomy unit. The numbers above the branches refer to bootstrap values (where greater than $50 \%$ support was obtained). Note the separate branches for strict aquaporins and aquaglyceroporins. The novel O. viverrini aquaporins belong to the latter assemblage, indicating that they can transport additional permants beyond water.

detected in all stages; however, highest expression was evident in the egg stage (Figure 3).

\section{Suppression of Ov-aqp gene expression}

$O v-a q p-1$ and $O v-a q p-2$ gene expression was suppressed in $O$. viverrini in vitro by introducing target-specific
dsRNAs using electroporation followed by soaking to mediate knockdown. Figure 4A shows the specific suppression of $O v-a q p-1$ by $99 \%, 57 \%, 72 \%, 83 \%$ and $91 \%$ measured on days 1, 36,10 and 16 after treatment with the dsRNAs, respectively. Figure $4 \mathrm{~B}$ shows the specific suppression of $O v-a q p-2$ by $58 \%, 92 \%$ and $77 \%$ measured 


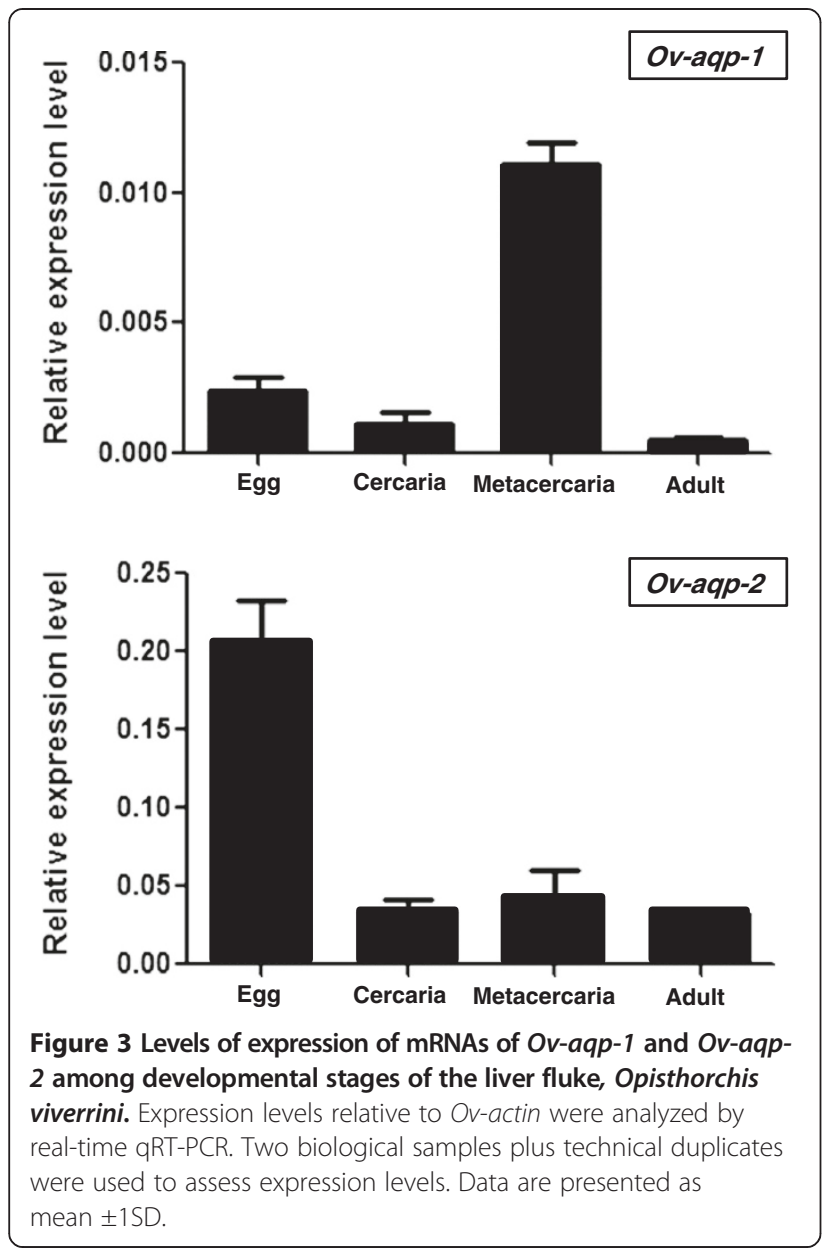

on days 1, 3 and 7 after treatment with the dsRNAs, respectively, compared to the negative control group that received luc dsRNA.

\section{Reduced Ov-AQP water transport in Ov-aqp dsRNA-treated flukes}

At the outset, to assess the capacity of adult O.viverrini to swell following prolonged incubation in distilled water, parasites were measured at intervals after transfer from physiological conditions in vitro (i.e. in RPMI) to hypotonic conditions (distilled water). This led to marked swelling of the worms by $10 \mathrm{~min}$, with the increase stabilizing by $30 \mathrm{~min}$ of incubation (Figure 5). The average size of worms at time $0 \mathrm{~min}$ was $10.5 \mathrm{~mm}^{2}$; after $5 \mathrm{~min}$ of incubation in water the average size of worms was $10.2 \mathrm{~mm}^{2}(p \leq 0.001)$ (Figure 5A). However, after $10 \mathrm{~min}$ of incubation in water, average worm size increased to $14.6 \mathrm{~mm}^{2}(p \leq 0.001)$ and $20.45 \mathrm{~mm}^{2}(p \leq 0.001)$ by $30 \mathrm{~min}$ of incubation. After $30 \mathrm{~min}$ in water, worms did not continue to swell further for the duration of the experiment (120 min). Based on these findings, further studies were carried out using the 10 min time point.

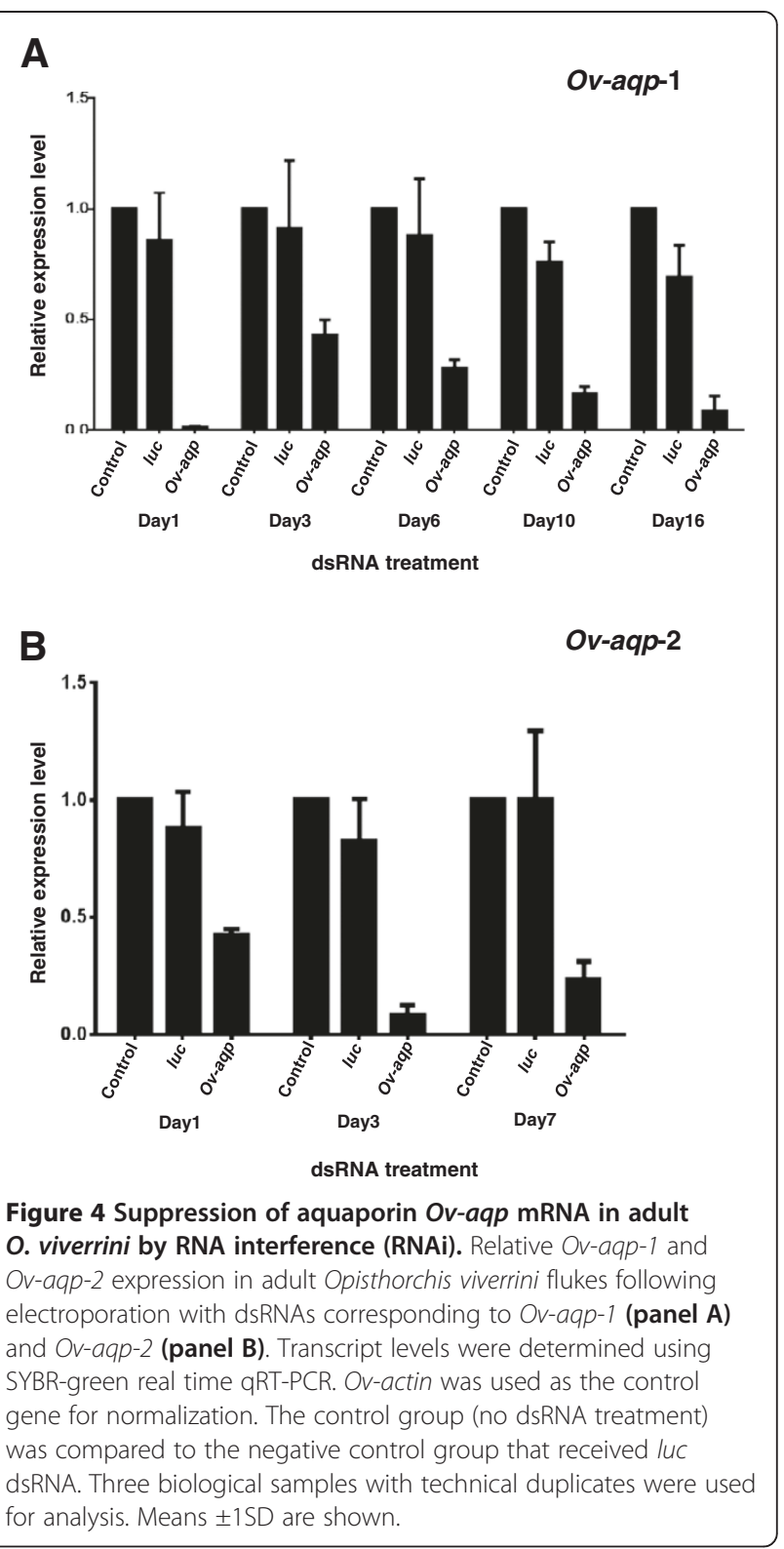

To determine whether silencing of $O v-a q p-1, O v-a q p-2$ and $O v-a q p-1+2$ expression affected water movement in $O$. viverrini, adult flukes treated with $O v$-aqp dsRNAs were transferred from RPMI into hypotonic medium as described above (distilled water). Parasites were photographed before and $10 \mathrm{~min}$ after transfer into water, and the sizes of a sample of individual parasites from control (mock control or $l u c$ ) and $O v$-aqp-suppressed groups were compared. When flukes were not subjected to osmotic shock by culture in RPMI medium, no significant difference in body size was detected between any of the control or dsRNA-treated flukes (range $=9.2-11.8 \mathrm{~mm}^{2}$; Figure 6A). When flukes were transferred into water, control flukes swelled to roughly twice their original size (in RPMI) with average sizes of $20.2 \mathrm{~mm}^{2}$ (mock control) 


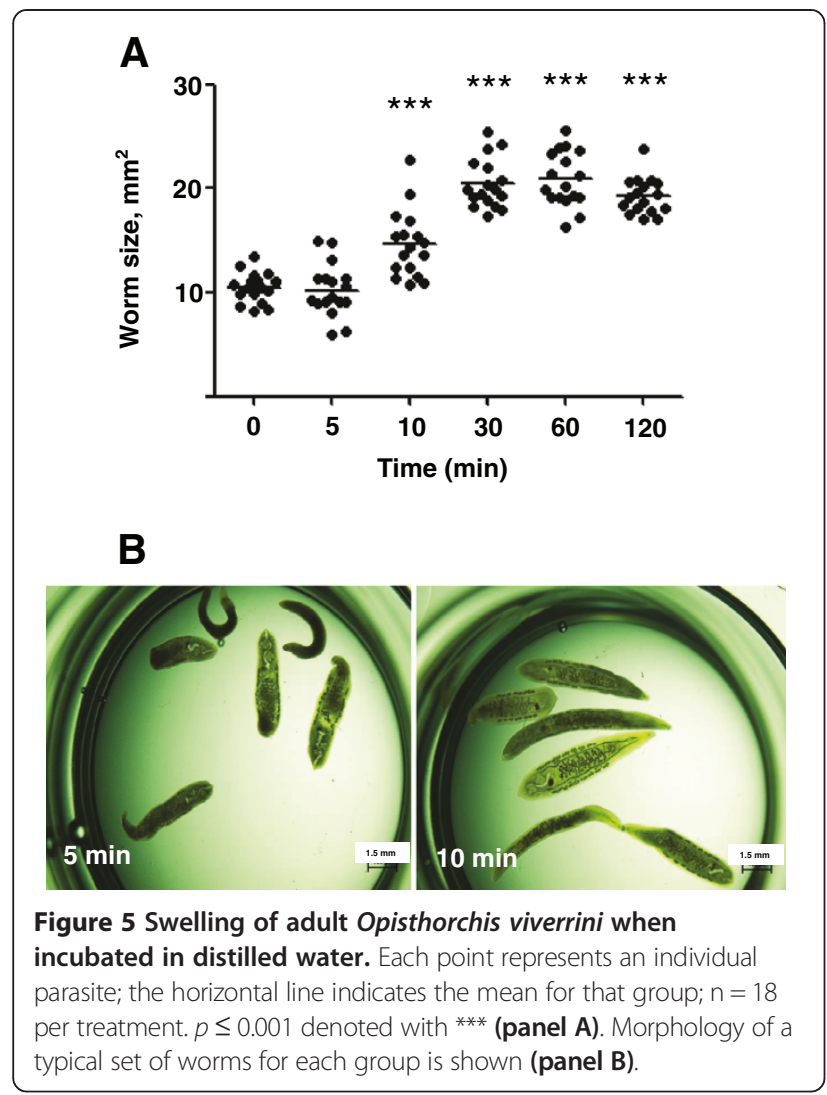

and $19.2 \mathrm{~mm}^{2}(l u c)$, however, flukes treated with $O v$-aqp1, $O v-a q p-2$ and $O v-a q p-1+2$ dsRNAs had significantly reduced average body sizes of15.55 $(p \leq 0.001), 16.05$ $(p \leq 0.001)$ and $16.3 \mathrm{~mm}^{2} \quad(p \leq 0.001)$, respectively (Figure 6B). The mean body sizes of mock and luc control groups were not significantly different.

Tissue sections of Ov-aqp-2 dsRNA-treated worms maintained in isotonic culture medium (RPMI) for 7 days showed that the parenchyma was more condensed than control worms (Figure 7), and parenchyma pore sizes were reduced in $O v$-aqp-2 dsRNA-treated worms (Figure 7).

\section{Discussion}

O. viverrini has a complex life cycle with each developmental stage inhabiting dissimilar environments. Freeliving stages including miracidia and cercariae live in fresh water for short periods before invading their respective snail and fish hosts. Parasitic stages including sporocysts, rediae, metacercariae and adult flukes live in the snail (sporocyst, redia), fish (metacercaria) and mammalian (adult fluke) hosts [31]. Like other eukaryotes, trematodes need to regulate water movement into and out of their cells. Transcriptomic and proteomic surveys of $O$. viverrini indicate abundant expression of a small number of AQPs, transporters that have been well characterized as water channels in other species $[16,17,22]$.

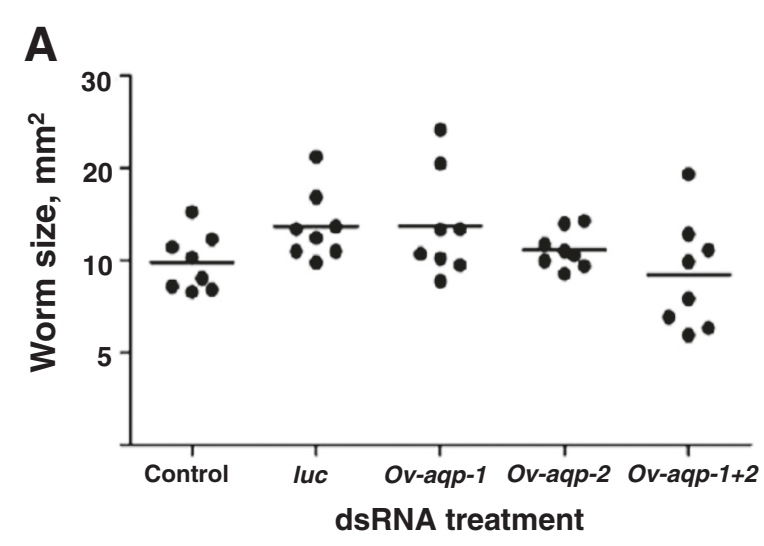

B

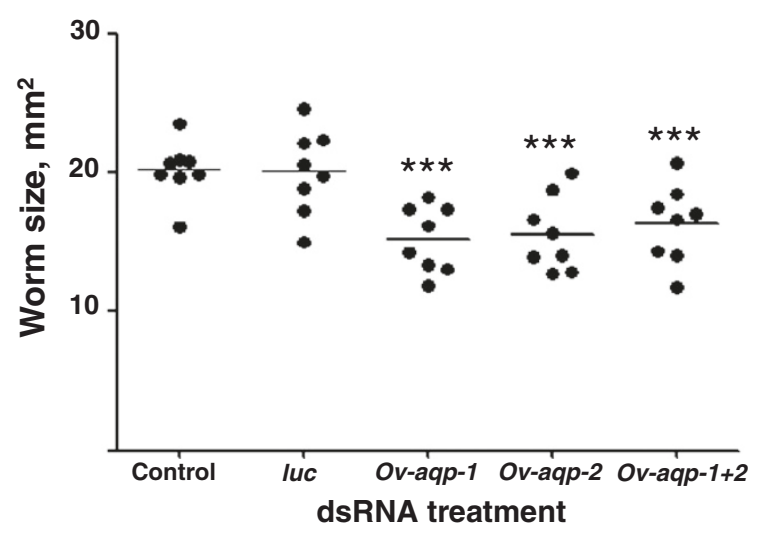

Figure 6 Suppression of Ov-aqp-1, Ov-aqp-2 and Ov-aqp-1+2 gene expression results in an inability to maintain osmotic flux. Size of cultured adult parasites after treatment with control (luc), Ov-aqp-1, Ov-aqp-2 and Ov-aqp-1 + 2dsRNAs when incubated in RPMI medium (A) or hypotonic medium (distilled water; B). Each point represents an individual parasite; horizontal line indicates the mean for that group; $n=8$ per treatment. $p \leq 0.001$ is indicated with ${ }^{* * *}$

In this study, cDNAs encoding aqp genes from $O$. viverrini were identified and cloned. AQP is a membrane integral protein that forms pores that facilitate passage of water and other small solutes through cell membranes. They function in cells where the membrane is intact and there is a need for a specific amount of water in the cell to maintain osmotic pressure. The structure of AQPs is well known [10]. Ov-AQP-1 and AQP-2 exhibited the typical AQP structure of six transmembrane domains with two NPA boxes that allow pore formation $[9,32]$.

The AQP family is comprised of three groups based on permeability properties. The first group contains the strict AQPs that allow only water to permeate the channel. Members of the second group conduct other small solutes, such as glycerol and urea; these transporters are termed aquaglyceroporins $[9,33]$. The third family of AQPs has a unique group of conserved residues surrounding the NPA 


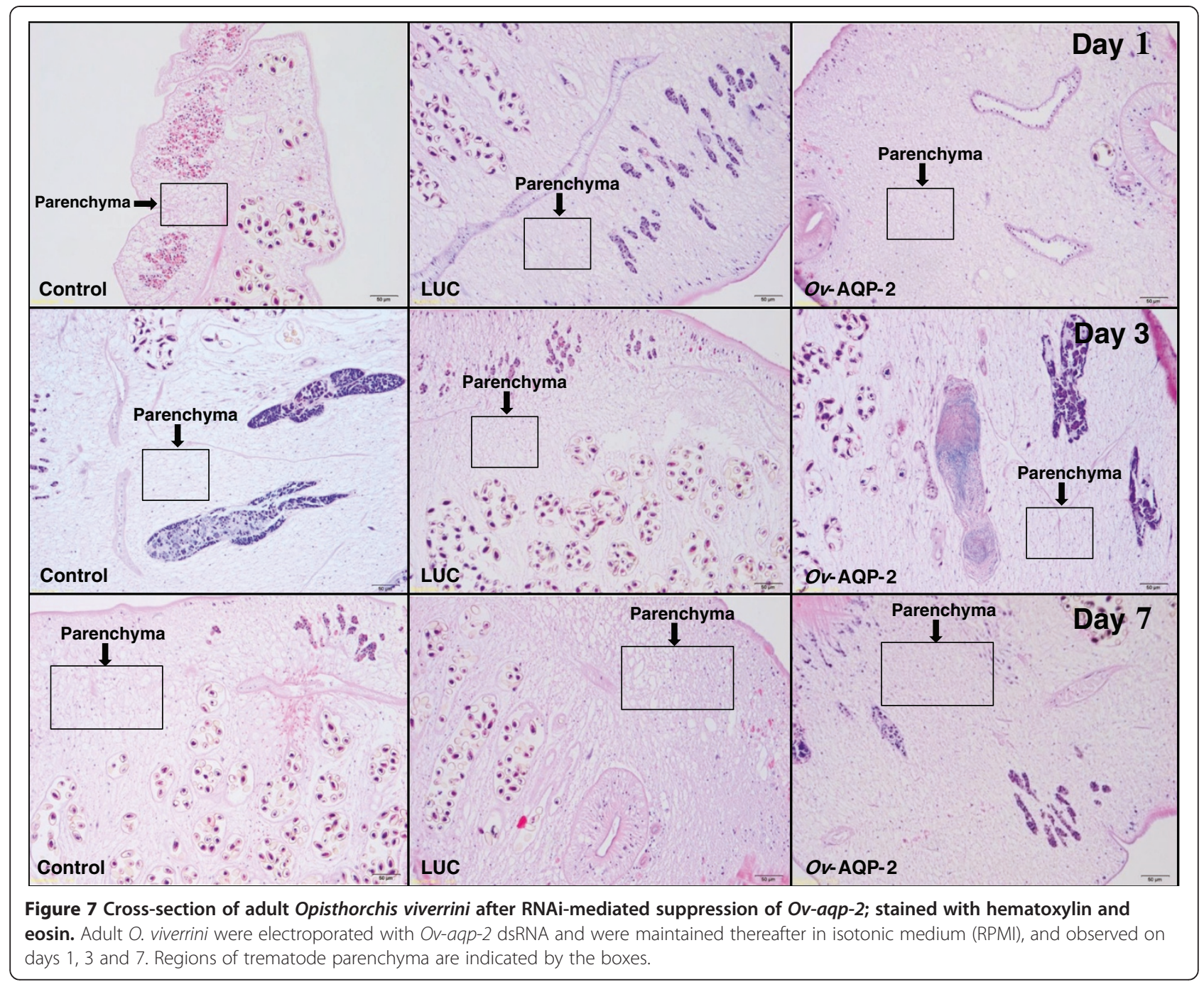

boxes; this group has numerous names including the $\mathrm{S}$-aquaporins (superaquaporins), aquaporins with unusual (or deviated) NPA boxes, and subcellular (or sip-like) aquaporins $[11,33,34]$. To date, the structures of nematode and trematode AQPs have generally consisted of six transmembrane domains and exhibited two NPA domaincontaining hemipores that overlap to form a pore between the membrane bilayer,a defining characteristic of AQPs $[13,35]$. Some aquaporins from the liver fluke Fasciola gigantica do not show conserved two NPA motifs, where the first NPA box is replaced by TAA although the second NPA box is conserved [36]. It is notable, therefore, that the O.viverrini AQPs reported here showed higher sequence similarity with the aquaglyceroporin group (human AQP3, -7, -9 and -10) $[9,10]$ as well as SmAQP from the human blood fluke, S. mansoni [12] and PfAQP from Plasmodium falciparum which exhibit a functional channel facilitating water and glycerol [37] compared to the traditional AQP group (human AQP-1, $-2,-5$ and -6) $[9,10]$, suggesting that permeants beyond water or glycerol might be transported into/from cells of $O$. viverrini.

Transcripts encoding aqp were detected in each of the developmental stages examined here, suggesting a requirement for these proteins throughout the entire life cycle of the parasite. The highest expression levels of $O v$-aqp-1 and $O v$-aqp-2 were detected in metacercariae and eggs, respectively, implying key roles in the physiology of the infectious process for AQP-1 in particular. To further address the functional roles of $\mathrm{Ov}$-AQPs, we used RNAi to suppress their expression and observed that both $O v-a q p-1$ and $a q p-2$ were essential for controlling osmosis in the adult fluke. Likewise, SmAQP is important for both entry and exit of water and other solutes in schistosomes [12]. Accordingly, AQP is being considered as a potential vaccine candidate for combating Schistosoma japonicum infections due to the presence of numerous B cell epitopes that are predicted to be accessible to host antibodies [38]. 
Histological analysis of $O v$-aqp-2 dsRNA-treated flukes showed that the average pore size in the parenchymal tissue was reduced compared to control worms. This finding, coupled with the inability of aqp-2-dsRNA treated worms to maintain osmotic pressure, implies that $\mathrm{Ov}$ AQP-2 is integral to entry and egress of water from the tissues of $O$. viverrini. Phylogenetic analysis revealed that the $O$. viverrini $\mathrm{AQPs}$ belong to the aquaglyceroporin group, suggesting that their roles extend to substrates beyond other small solutes $[37,39,40]$. However, in this study we investigated only regulation of water into the body wall. Several authors have investigated other functions of AQPs in schistosomes, including the entry/exit of drugs, highlighting the importance of the trematode tegument in osmoregulation, and the uptake and excretion of metabolic waste and drugs [12,14]. In like fashion, $\mathrm{O} v$-AQPs might play similar roles in drug uptake and waste excretion, and we propose to explore these potential functions in the future. In conclusion, AQPs likely perform essential roles in the movement of water between the external environment and the liver fluke. Drugs that counteract mammalian AQPs are in development [41,42], and accordingly liver fluke AQPs represent potential new targets for interventions to control and treat this carcinogenic pathogen.

\section{Conclusion}

Aquaporins (AQPs) belong to the major intrinsic protein superfamily of integral plasma membrane channel proteins that selectively transport water across cell membranes. AQPs play key roles as water and ion transport channels through the tegument of trematodes. Here we investigated two discrete forms of AQP mRNAs from the adult $O$. viverrini. Phylogenetic analysis indicated that $\mathrm{Ov}$-AQPs belong to aquaglyceroporin-like water channel proteins characterized from parasites, which may be integral to the specialized requirements of water and solute control during parasitism. Ov-aqps transcripts were detected in all developmental stages of $O$. viverrini. RNA interference using electroporated dsRNA suppressed transcript levels of Ov-aqps in adult worms by $58-99 \%$, and disabled water transport in adult flukes.The apparently pivotal roles of $\mathrm{Ov}$-AQP in solute homeostasis at the fluke surface suggest that deeper investigation will be informative for the pathophysiology of O. viverrini, and may uncover intervention targets, particularly in view of the singularly notable predilection of this pathogen for residence within the biliary tree.

\section{Competing interests}

The authors declare that they have no competing interests.

\section{Authors' contributions}

ST, PJB, AL and TL conceived and designed the study. ST and SP performed experiments. ST, SK and TL performed data analysis and interpretation. ST, PJB, AL and TL wrote the manuscript. All authors reviewed the draft and accepted it for submission. All authors read and approved the final version of the manuscript.

\section{Acknowledgements}

This work was supported by the Higher Education Research Promotion and National Research University Project of Thailand, Office of the Higher

Education Commission, Grant under the program Strategic Scholarships for Frontier Research Network for the Ph.D. Program Thai Doctoral degree from the Office of the Higher Education Commission, Thailand and from the National Institute of Allergy and Infectious Disease (NIAID) of the United States National Institutes of Health (NIH) award number P50Al098639 (Tropical Medicine Research Center). The content is solely the responsibility of the authors and does not necessarily represent the official views of the NIAID or the NIH.AL is supported by a NHMRC principal research fellowship.

\section{Author details}

${ }^{1}$ Department of Parasitology, Faculty of Medicine, Khon Kaen University, Khon Kaen 40002, Thailand. '2Department of Microbiology, Immunology and Tropical Medicine, Research Center for Neglected Diseases of Poverty, School of Medicine \& Health Sciences, George Washington University, Washington, DC 20037, USA. ${ }^{3}$ Centre for Biodiscovery and Molecular Development of Therapeutics, Australian Institute of Tropical Health and Medicine, James Cook University, Cairns, Queensland 4878, Australia. ${ }^{4}$ Liver Fluke and Cholangiocarcinoma Research Center, Faculty of Medicine, Khon Kaen University, Khon Kaen 40002, Thailand.

Received: 18 March 2014 Accepted: 9 May 2014

Published: 14 May 2014

\section{References}

1. Sithithaworn $P$, Andrews RH, Nguyen VD, Wongsaroj T, Sinuon M, Odermatt P, Nawa Y, Liang S, Brindley PJ, Sripa B: The current status of opisthorchiasis and clonorchiasis in the Mekong Basin. Parasitol Int 2012, 61(1):10-16.

2. Sripa B: Global burden of food-borne trematodiasis. Lancet Infect Dis 2012, 12(3):171-172

3. Sripa B, Brindley PJ, Mulvenna J, Laha T, Smout MJ, Mairiang E, Bethony JM, Loukas A: The tumorigenic liver fluke Opisthorchis viverrini-multiple pathways to cancer. Trends Parasitol 2012, 28(10):395-407.

4. Geary TG, Woods DJ, Williams T, Nwaka S: Target identification and mechanism-based screening for anthelmintics: Application of veterinary antiparasitic research programmes to search for new antiparasitic drugs for human indications. In Antiparasitic and Antibacterial Drug Discovery: From Molecular Targets to Drug Candidates. Edited by Selzer PM. Weinheim: Wiley-Blackwell; 2009:3-15.

5. Taylor CM, Wang Q, Rosa BA, Huang SC, Powell K, Schedl T, Pearce EJ, Abubucker S, Mitreva M: Discovery of anthelmintic drug targets and drugs using chokepoints in nematode metabolic pathways. PLOS Pathog 2013, 9(8):e1003505.

6. Kaminsky R, Ducray P, Jung M, Clover R, Rufener L, Bouvier J, Weber SS, Wenger A, Wieland-Berghausen S, Goebel T, Gauvry N, Pautrat F, Skripsky T, Froelich O, Komoin-Oka C, Westlund B, Sluder A, Maser P: A new class of anthelmintics effective against drug-resistant nematodes. Nature 2008 452(7184):176-180.

7. Beitz E: Aquaporins from pathogenic protozoan parasites: structure, function and potential for chemotherapy. Biol Cell 2005, 97(6):373-383.

8. Zeuthen T: How water molecules pass through aquaporins. Trends Biochem Sci 2001, 26(2):77-79.

9. Zardoya R: Phylogeny and evolution of the major intrinsic protein family. Biol Cell 2005, 97(6):397-414.

10. Verkman AS, Mitra AK: Structure and function of aquaporin water channels. Am J Physiol Renal Physiol 2000, 278(1):F13-F28.

11. Ishibashi K: Aquaporin subfamily with unusual NPA boxes. Biochim Biophys Acta 2006, 1758(8):989-993.

12. Faghiri Z, Skelly PJ: The role of tegumental aquaporin from the human parasitic worm, Schistosoma mansoni, in osmoregulation and drug uptake. Faseb J 2009, 23(8):2780-2789.

13. Song J, Mak E, Wu B, Beitz E: Parasite aquaporins: Current developments in drug facilitation and resistance. Biochim Biophys Acta 2014, 1840(5):1566-1573. 
14. Faghiri Z, Camargo SM, Huggel K, Forster IC, Ndegwa D, Verrey F, Skelly PJ: The tegument of the human parasitic worm Schistosoma mansoni as an excretory organ: the surface aquaporin SmAQP is a lactate transporter. PLoS One 2010, 5(5):e10451.

15. Da'dara A, Krautz-Peterson G, Faghiri Z, Skelly PJ: Metabolite movement across the schistosome surface. J Helminthol 2012, 86(2):141-147.

16. Laha T, Pinlaor P, Mulvenna J, Sripa B, Sripa M, Smout MJ, Gasser RB, Brindley PJ, Loukas A: Gene discovery for the carcinogenic human liver fluke, Opisthorchis viverrini. BMC Genomics 2007, 8:189.

17. Mulvenna J, Sripa B, Brindley PJ, Gorman J, Jones MK, Colgrave ML, Jones A Nawaratna S, Laha T, Suttiprapa S, Smout MJ, Loukas A: The secreted and surface proteomes of the adult stage of the carcinogenic human liver fluke Opisthorchis viverrini. Proteomics 2010, 10(5):1063-1078.

18. Sithithaworn P, Pipitgool V, Srisawangwong T, Elkins DB, Haswell-Elkins MR: Seasonal variation of Opisthorchis viverrini infection in cyprinoid fish in north-east Thailand: implications for parasite control and food safety. Bull World Health Organ 1997, 75(2):125-131.

19. Sripa B, Kaewkes S: Gall bladder and extrahepatic bile duct changes in Opisthorchis viverrini-infected hamsters. Acta Trop 2002, 83(1):29-36.

20. Piratae S, Tesana S, Jones MK, Brindley PJ, Loukas A, Lovas E, Eursitthichai V, Sripa B, Thanasuwan S, Laha T: Molecular characterization of a tetraspanin from the human liver fluke, Opisthorchis viverrini. PLoS Negl Trop Dis 2012, 6(12):e1939.

21. Kaewkes S, Kaewkes W, Boonmars T, Sripa B: Effect of light intensity on Opisthorchis viverrini cercarial shedding levels from Bithynia snails-a preliminary study. Parasitol Int 2012, 61(1):46-48.

22. Young ND, Campbell BE, Hall RS, Jex AR, Cantacessi C, Laha T, Sohn WM, Sripa B, Loukas A, Brindley PJ, Gasser RB: Unlocking the transcriptomes of two carcinogenic parasites, Clonorchis sinensis and Opisthorchis viverrini. PLoS Negl Trop Dis 2010, 4(6):e719.

23. Altschul SF, Madden TL, Schaffer AA, Zhang J, Zhang Z, Miller W, Lipman DJ: Gapped BLAST and PSI-BLAST: a new generation of protein database search programs. Nucleic Acids Res 1997, 25(17):3389-3402.

24. Hall T: BioEdit is a biological sequence alignment editor written for Windows 95/98/NT/2000/XP. Carlsbad, CA: Nucl Acids Symp Ser; 1999.

25. Saitou N, Nei M: The neighbor-joining method: a new method for reconstructing phylogenetic trees. Mol Biol Evol 1987, 4(4):406-425.

26. Tamura K, Stecher G, Peterson D, Filipski A, Kumar S: MEGA6: Molecular Evolutionary Genetics Analysis version 6.0. Mol Biol Evol 2013, 30(12):2725-2729.

27. Schmittgen TD, Livak KJ: Analyzing real-time PCR data by the comparative C(T) method. Nat Protoc 2008, 3(6):1101-1108.

28. Rinaldi G, Morales ME, Cancela M, Castillo E, Brindley PJ, Tort JF: Development of functional genomic tools in trematodes: RNA interference and luciferase reporter gene activity in Fasciola hepatica. PLoS Negl Trop Dis 2008, 2(7):e260.

29. Sripa B, Kaewkes S: Localisation of parasite antigens and inflammatory responses in experimental opisthorchiasis. Int J Parasitol 2000, 30(6):735-740.

30. Wang X, Chen W, Huang Y, Sun J, Men J, Liu H, Luo F, Guo L, Lv X, Deng C, Zhou C, Fan Y, Li X, Huang L, Hu Y, Liang C, Hu X, Xu J, Yu X: The draft genome of the carcinogenic human liver fluke Clonorchis sinensis. Genome Biol 2011, 12(10):R107.

31. Wykoff DE, Harinasuta C, Juttijudata P, Winn MM: Opisthorchis viverrini in Thailand-the Life Cycle and Comparison with O. Felineus. J Parasitol 1965, 51:207-214

32. Benga G: The first discovered water channel protein, later called aquaporin 1: molecular characteristics, functions and medical implications. Mol Aspects Med 2012, 33(5-6):518-534

33. Benga $\mathrm{G}$ : On the definition, nomenclature and classification of water channel proteins (aquaporins and relatives). Mol Aspects Med 2012 33(5-6):514-517.

34. Ishibashi K: Aquaporin superfamily with unusual npa boxes: S-aquaporins (superfamily, sip-like and subcellular-aquaporins). Cell Mol Biol 2006, 52(7):20-27.

35. Loukas A, Hunt P, Maizels RM: Cloning and expression of an aquaporinlike gene from a parasitic nematode. Mol Biochem Parasitol 1999, 99(2):287-293.

36. Geadkaew A, Von Bulow J, Beitz E, Grams SV, Viyanant V, Grams R: Functional analysis of novel aquaporins from Fasciola gigantica. Mol Biochem Parasitol 2011, 175(2):144-153.
37. Hansen M, Kun JF, Schultz JE, Beitz E: A single, bi-functional aquaglyceroporin in blood-stage Plasmodium falciparum malaria parasites. J Biol Chem 2002, 277(7):4874-4882.

38. Song J, He QF: Bioinformatics analysis of the structure and linear B-cell epitopes of aquaporin-3 from Schistosoma japonicum. Asian Pac J Trop Med 2012, 5(2):107-109.

39. Liu F, Lu J, Hu W, Wang SY, Cui SJ, Chi M, Yan Q, Wang XR, Song HD, Xu XN, Wang JJ, Zhang XL, Zhang X, Wang ZQ, Xue CL, Brindley PJ, McManus DP, Yang PY, Feng Z, Chen Z, Han ZG: New perspectives on host-parasite interplay by comparative transcriptomic and proteomic analyses of Schistosoma japonicum. PLoS Pathog 2006, 2(4):e29.

40. Bassarak B, Uzcategui NL, Schonfeld C, Duszenko M: Functional characterization of three aquaglyceroporins from Trypanosoma brucei in osmoregulation and glycerol transport. Cell Physiol Biochem 2011, 27(3-4):411-420.

41. Wacker SJ, Aponte-Santamaria C, Kjellbom P, Nielsen S, De Groot BL, Rutzler $M$ : The identification of novel, high affinity AQP9 inhibitors in an intracellular binding site. Mol Membr Biol 2013, 30(3):246-260.

42. Martins AP, Marrone A, Ciancetta A, Galan Cobo A, Echevarria M, Moura TF, Re N, Casini A, Soveral G: Targeting aquaporin function: potent inhibition of aquaglyceroporin-3 by a gold-based compound. PLoS One 2012, 7(5):e37435.

doi:10.1186/1756-3305-7-224

Cite this article as: Thanasuwan et al: Suppression of aquaporin, a mediator of water channel control in the carcinogenic liver fluke, Opisthorchis viverrini. Parasites \& Vectors 2014 7:224.

\section{Submit your next manuscript to BioMed Central and take full advantage of:}

- Convenient online submission

- Thorough peer review

- No space constraints or color figure charges

- Immediate publication on acceptance

- Inclusion in PubMed, CAS, Scopus and Google Scholar

- Research which is freely available for redistribution

Submit your manuscript at www.biomedcentral.com/submit
C) Biomed Central 